\title{
"Phasic jaw motor episodes in healthy subjects with or without clinical signs and symptoms of sleep bruxism: a pilot study". A critical commentary
}

\author{
Ephraim Winocur
}

Received: 29 April 2013 / Accepted: 22 May 2013 / Published online: 4 June 2013

(C) Springer-Verlag Berlin Heidelberg 2013

Sleep bruxism (SB) is a frustrating phenomenon. Its pathophysiology is still controversial, there is no definitive effective treatment, its clinical diagnosis is unreliable, and the gold standard accepted laboratory diagnosis (polysomnographic evaluation) difficult and expensive. SB is often detrimental to natural dentition and may be the direct reason for failures of dental restorative treatments and dental implants. Thus, early clinical diagnosis is often crucial for the success of a dental rehabilitation.

Due to the scarcity of reliable and valid diagnostic tools for bruxism, an international group of bruxism experts [1], proposed a diagnostic grading system for clinical and research purposes. The international group suggested several diagnostic categories: (1) "possible" sleep or awake bruxism — diagnosis based on self-report through questionnaires and/or the anamnestic part of a clinical examination; (2) "Probable" sleep or awake bruxism - diagnosis based on self-report plus clinical inspection; (3) "Definite" sleep bruxism — based on self-report, clinical examination, and a polysomnographic recording, preferably along with audio/video recordings. According to these definitions the definitive gold standard for sleep bruxism diagnosis is polysomnographic evaluations. However, large sample studies on bruxism can be performed only by adopting clinical diagnostic methods, generally based on questionnaires. Lavigne et al. [2] pointed out that questionnaires may be subject to bias, such as the natural fluctuation in bruxism motor activity over time, the risk of poor or imprecise recall regarding bruxism or anxiety and the lack of awareness of the current behavior. In a recently published paper [3], we discussed the

\section{E. Winocur $(\square)$}

Department of Oral Rehabilitation The Maurice and Gabriela

Goldschleger School of Dental Medicine, Tel Aviv University,

Tel Aviv 69978, Israel

e-mail: winocur@post.tau.ac.il different methods for bruxism diagnosis with the conclusion that the use of self-report questionnaires is inevitable when investigating large population samples. The recommendation was that the validity of such questionnaires studies needs to be further evaluated through comparisons to polysomnographic study. The issue of accuracy and reliability of clinical or self-perceived bruxism versus instrumentally detected bruxism remain open, since it has never been tested scientifically. In other words, can a clinician depend on clinical diagnosis of SB before starting an extensive dental rehabilitation? What's more, is a diagnosis of possible, or even probable, bruxism reliable enough in clinical practice? This is exactly the aim of the study by Yoshizawa et al. [4] in this issue. They investigated the association between clinical diagnostic criteria for SB, and the frequency of jaw motor events during sleep. Their results partially illuminate the open questions asked above. They found that those subjects with a report of tooth grinding and tooth attrition had a higher frequency of rhythmic masticatory muscle activity episodes during sleep than those without, while the presence or absence of morning masticatory muscle symptoms and masseter muscle hypertrophy failed to reveal a difference in the frequency of jaw motor episodes during sleep. The authors hypothesized that these data may suggest the possibility that the clinical signs and symptoms tested in their study could represent distinct clinical and physiological components of sleep jaw motor activity during sleep. This hypothesis is of great clinical importance, since it may guide the clinician before deciding which the best treatment for the patient is. The discussion of this article is very illustrative and contains an overview of the available studies on the same subject. The study limitations and the clinical implications are well described in the article and should be taken into consideration before adopting the conclusions of this pioneer study. 


\section{References}

1. Lobbezoo F, Ahlberg J, Glaros AG, Kato T, Koyano K, Lavigne GJ, de Leeuw R, Manfredini D, Svensson P, Winocur E (2013) Bruxism defined and graded: an international consensus. J Oral Rehabil 40:2-4

2. Lavigne GJ, Khoury S, Abe S, Yamaguchi T, Raphael K (2008) Bruxism physiology and pathology: an overview for clinicians. J Oral Rehabil 35:476-494
3. Winocur E, Uziel N, Lisha T, Goldsmith C, Eli I (2011) Sleep and awake bruxism: associations with perceived stress, motivation for control, dental anxiety and gagging. J Oral Rehabil 38:3-11

4. Yoshizawa S, Suganuma T, Takaba M, Ono Y, Sakai T, Yoshizawa A, Kawana F, Kato T, Baba K: Phasic jaw motor episodes in healthy subjects with or without clinical signs and symptoms of sleep bruxism: a pilot study. Sleep and Breathing; in press 